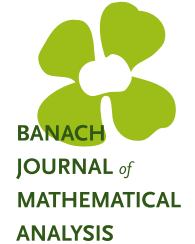

Banach J. Math. Anal. 10 (2016), no. 2, 385-399

http://dx.doi.org/10.1215/17358787-3495693

ISSN: $1735-8787$ (electronic)

http://projecteuclid.org/bjma

\title{
ON THE POWERS OF MAXIMAL IDEALS IN THE MEASURE ALGEBRA
}

\author{
LÁSZLÓ SZÉKELYHIDI \\ Communicated by C. Badea
}

\begin{abstract}
In this paper, we describe the powers of maximal ideals in the measure algebra of some locally compact Abelian groups in terms of the derivatives of the Fourier-Laplace transform of compactly supported measures. We show that if the locally compact Abelian group has sufficiently many real characters, then all derivatives of the Fourier-Laplace transform of a measure at some point of its spectrum completely characterize the measure. We also show that the derivatives of the Fourier-Laplace transform of a measure can be used to describe the powers of the maximal ideals corresponding to the points of the spectrum of the measure on discrete Abelian groups with finite torsion-free rank.
\end{abstract}

\section{INTRODUCTION}

Spectral analysis and spectral synthesis deal with the description of different varieties. One of the fundamental theorems about spectral synthesis in this field is due to Laurent Schwartz. Recently, several new results on spectral analysis and spectral synthesis have been found on discrete Abelian groups (see [6], [7], [5]) and also in the nondiscrete case (see, e.g., [3], [8]-[10]). In [2], the author formulated problems and proved results concerning spectral synthesis on locally compact Abelian groups. Recently, we introduced a method of studying spectral synthesis problems using annihilators of varieties on locally compact Abelian groups (see [11]). Based on these investigations, it has turned out that the powers of maximal ideals in the group algebra of a discrete Abelian group, or, more generally, in the

Copyright 2016 by the Tusi Mathematical Research Group.

Received Apr. 14, 2015; Accepted Jul. 11, 2015.

2010 Mathematics Subject Classification. Primary 43A25; Secondary 43A45, 22 D15.

Keywords. group algebra, spectral analysis, spectral synthesis, annihilator, exponential monomial. 


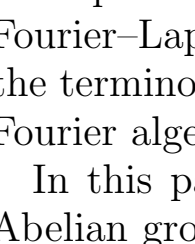

Banach J. Math. Anal. 10 (2016), no. 2, 385-399

http://dx.doi.org/10.1215/17358787-3495693

ISSN: $1735-8787$ (electronic)

http://projecteuclid.org/bjma

\title{
ON THE POWERS OF MAXIMAL IDEALS IN THE MEASURE ALGEBRA
}

\author{
LÁSZLÓ SZÉKELYHIDI \\ Communicated by C. Badea
}

\begin{abstract}
In this paper, we describe the powers of maximal ideals in the measure algebra of some locally compact Abelian groups in terms of the derivatives of the Fourier-Laplace transform of compactly supported measures. We show that if the locally compact Abelian group has sufficiently many real characters, then all derivatives of the Fourier-Laplace transform of a measure at some point of its spectrum completely characterize the measure. We also show that the derivatives of the Fourier-Laplace transform of a measure can be used to describe the powers of the maximal ideals corresponding to the points of the spectrum of the measure on discrete Abelian groups with finite torsion-free rank.
\end{abstract}

\section{INTRODUCTION}

Spectral analysis and spectral synthesis deal with the description of different varieties. One of the fundamental theorems about spectral synthesis in this field is due to Laurent Schwartz. Recently, several new results on spectral analysis and spectral synthesis have been found on discrete Abelian groups (see [6], [7], [5]) and also in the nondiscrete case (see, e.g., [3], [8]-[10]). In [2], the author formulated problems and proved results concerning spectral synthesis on locally compact Abelian groups. Recently, we introduced a method of studying spectral synthesis problems using annihilators of varieties on locally compact Abelian groups (see [11]). Based on these investigations, it has turned out that the powers of maximal ideals in the group algebra of a discrete Abelian group, or, more generally, in the

Copyright 2016 by the Tusi Mathematical Research Group.

Received Apr. 14, 2015; Accepted Jul. 11, 2015.

2010 Mathematics Subject Classification. Primary 43A25; Secondary 43A45, 22 D15.

Keywords. group algebra, spectral analysis, spectral synthesis, annihilator, exponential monomial. 


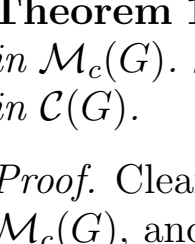

Banach J. Math. Anal. 10 (2016), no. 2, 385-399

http://dx.doi.org/10.1215/17358787-3495693

ISSN: $1735-8787$ (electronic)

http://projecteuclid.org/bjma

\title{
ON THE POWERS OF MAXIMAL IDEALS IN THE MEASURE ALGEBRA
}

\author{
LÁSZLÓ SZÉKELYHIDI \\ Communicated by C. Badea
}

\begin{abstract}
In this paper, we describe the powers of maximal ideals in the measure algebra of some locally compact Abelian groups in terms of the derivatives of the Fourier-Laplace transform of compactly supported measures. We show that if the locally compact Abelian group has sufficiently many real characters, then all derivatives of the Fourier-Laplace transform of a measure at some point of its spectrum completely characterize the measure. We also show that the derivatives of the Fourier-Laplace transform of a measure can be used to describe the powers of the maximal ideals corresponding to the points of the spectrum of the measure on discrete Abelian groups with finite torsion-free rank.
\end{abstract}

\section{INTRODUCTION}

Spectral analysis and spectral synthesis deal with the description of different varieties. One of the fundamental theorems about spectral synthesis in this field is due to Laurent Schwartz. Recently, several new results on spectral analysis and spectral synthesis have been found on discrete Abelian groups (see [6], [7], [5]) and also in the nondiscrete case (see, e.g., [3], [8]-[10]). In [2], the author formulated problems and proved results concerning spectral synthesis on locally compact Abelian groups. Recently, we introduced a method of studying spectral synthesis problems using annihilators of varieties on locally compact Abelian groups (see [11]). Based on these investigations, it has turned out that the powers of maximal ideals in the group algebra of a discrete Abelian group, or, more generally, in the

Copyright 2016 by the Tusi Mathematical Research Group.

Received Apr. 14, 2015; Accepted Jul. 11, 2015.

2010 Mathematics Subject Classification. Primary 43A25; Secondary 43A45, 22 D15.

Keywords. group algebra, spectral analysis, spectral synthesis, annihilator, exponential monomial. 


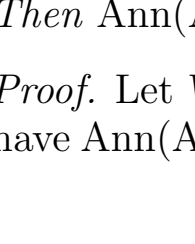

Banach J. Math. Anal. 10 (2016), no. 2, 385-399

http://dx.doi.org/10.1215/17358787-3495693

ISSN: $1735-8787$ (electronic)

http://projecteuclid.org/bjma

\title{
ON THE POWERS OF MAXIMAL IDEALS IN THE MEASURE ALGEBRA
}

\author{
LÁSZLÓ SZÉKELYHIDI \\ Communicated by C. Badea
}

\begin{abstract}
In this paper, we describe the powers of maximal ideals in the measure algebra of some locally compact Abelian groups in terms of the derivatives of the Fourier-Laplace transform of compactly supported measures. We show that if the locally compact Abelian group has sufficiently many real characters, then all derivatives of the Fourier-Laplace transform of a measure at some point of its spectrum completely characterize the measure. We also show that the derivatives of the Fourier-Laplace transform of a measure can be used to describe the powers of the maximal ideals corresponding to the points of the spectrum of the measure on discrete Abelian groups with finite torsion-free rank.
\end{abstract}

\section{INTRODUCTION}

Spectral analysis and spectral synthesis deal with the description of different varieties. One of the fundamental theorems about spectral synthesis in this field is due to Laurent Schwartz. Recently, several new results on spectral analysis and spectral synthesis have been found on discrete Abelian groups (see [6], [7], [5]) and also in the nondiscrete case (see, e.g., [3], [8]-[10]). In [2], the author formulated problems and proved results concerning spectral synthesis on locally compact Abelian groups. Recently, we introduced a method of studying spectral synthesis problems using annihilators of varieties on locally compact Abelian groups (see [11]). Based on these investigations, it has turned out that the powers of maximal ideals in the group algebra of a discrete Abelian group, or, more generally, in the

Copyright 2016 by the Tusi Mathematical Research Group.

Received Apr. 14, 2015; Accepted Jul. 11, 2015.

2010 Mathematics Subject Classification. Primary 43A25; Secondary 43A45, 22 D15.

Keywords. group algebra, spectral analysis, spectral synthesis, annihilator, exponential monomial. 


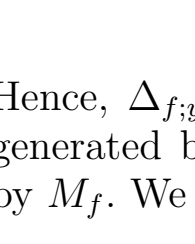

Banach J. Math. Anal. 10 (2016), no. 2, 385-399

http://dx.doi.org/10.1215/17358787-3495693

ISSN: $1735-8787$ (electronic)

http://projecteuclid.org/bjma

\title{
ON THE POWERS OF MAXIMAL IDEALS IN THE MEASURE ALGEBRA
}

\author{
LÁSZLÓ SZÉKELYHIDI \\ Communicated by C. Badea
}

\begin{abstract}
In this paper, we describe the powers of maximal ideals in the measure algebra of some locally compact Abelian groups in terms of the derivatives of the Fourier-Laplace transform of compactly supported measures. We show that if the locally compact Abelian group has sufficiently many real characters, then all derivatives of the Fourier-Laplace transform of a measure at some point of its spectrum completely characterize the measure. We also show that the derivatives of the Fourier-Laplace transform of a measure can be used to describe the powers of the maximal ideals corresponding to the points of the spectrum of the measure on discrete Abelian groups with finite torsion-free rank.
\end{abstract}

\section{INTRODUCTION}

Spectral analysis and spectral synthesis deal with the description of different varieties. One of the fundamental theorems about spectral synthesis in this field is due to Laurent Schwartz. Recently, several new results on spectral analysis and spectral synthesis have been found on discrete Abelian groups (see [6], [7], [5]) and also in the nondiscrete case (see, e.g., [3], [8]-[10]). In [2], the author formulated problems and proved results concerning spectral synthesis on locally compact Abelian groups. Recently, we introduced a method of studying spectral synthesis problems using annihilators of varieties on locally compact Abelian groups (see [11]). Based on these investigations, it has turned out that the powers of maximal ideals in the group algebra of a discrete Abelian group, or, more generally, in the

Copyright 2016 by the Tusi Mathematical Research Group.

Received Apr. 14, 2015; Accepted Jul. 11, 2015.

2010 Mathematics Subject Classification. Primary 43A25; Secondary 43A45, 22 D15.

Keywords. group algebra, spectral analysis, spectral synthesis, annihilator, exponential monomial. 


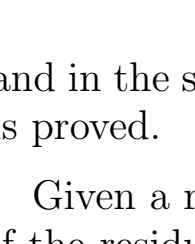

Banach J. Math. Anal. 10 (2016), no. 2, 385-399

http://dx.doi.org/10.1215/17358787-3495693

ISSN: $1735-8787$ (electronic)

http://projecteuclid.org/bjma

\title{
ON THE POWERS OF MAXIMAL IDEALS IN THE MEASURE ALGEBRA
}

\author{
LÁSZLÓ SZÉKELYHIDI \\ Communicated by C. Badea
}

\begin{abstract}
In this paper, we describe the powers of maximal ideals in the measure algebra of some locally compact Abelian groups in terms of the derivatives of the Fourier-Laplace transform of compactly supported measures. We show that if the locally compact Abelian group has sufficiently many real characters, then all derivatives of the Fourier-Laplace transform of a measure at some point of its spectrum completely characterize the measure. We also show that the derivatives of the Fourier-Laplace transform of a measure can be used to describe the powers of the maximal ideals corresponding to the points of the spectrum of the measure on discrete Abelian groups with finite torsion-free rank.
\end{abstract}

\section{INTRODUCTION}

Spectral analysis and spectral synthesis deal with the description of different varieties. One of the fundamental theorems about spectral synthesis in this field is due to Laurent Schwartz. Recently, several new results on spectral analysis and spectral synthesis have been found on discrete Abelian groups (see [6], [7], [5]) and also in the nondiscrete case (see, e.g., [3], [8]-[10]). In [2], the author formulated problems and proved results concerning spectral synthesis on locally compact Abelian groups. Recently, we introduced a method of studying spectral synthesis problems using annihilators of varieties on locally compact Abelian groups (see [11]). Based on these investigations, it has turned out that the powers of maximal ideals in the group algebra of a discrete Abelian group, or, more generally, in the

Copyright 2016 by the Tusi Mathematical Research Group.

Received Apr. 14, 2015; Accepted Jul. 11, 2015.

2010 Mathematics Subject Classification. Primary 43A25; Secondary 43A45, 22 D15.

Keywords. group algebra, spectral analysis, spectral synthesis, annihilator, exponential monomial. 


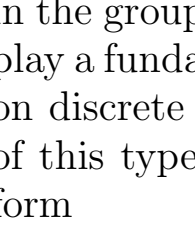

Banach J. Math. Anal. 10 (2016), no. 2, 385-399

http://dx.doi.org/10.1215/17358787-3495693

ISSN: $1735-8787$ (electronic)

http://projecteuclid.org/bjma

\title{
ON THE POWERS OF MAXIMAL IDEALS IN THE MEASURE ALGEBRA
}

\author{
LÁSZLÓ SZÉKELYHIDI \\ Communicated by C. Badea
}

\begin{abstract}
In this paper, we describe the powers of maximal ideals in the measure algebra of some locally compact Abelian groups in terms of the derivatives of the Fourier-Laplace transform of compactly supported measures. We show that if the locally compact Abelian group has sufficiently many real characters, then all derivatives of the Fourier-Laplace transform of a measure at some point of its spectrum completely characterize the measure. We also show that the derivatives of the Fourier-Laplace transform of a measure can be used to describe the powers of the maximal ideals corresponding to the points of the spectrum of the measure on discrete Abelian groups with finite torsion-free rank.
\end{abstract}

\section{INTRODUCTION}

Spectral analysis and spectral synthesis deal with the description of different varieties. One of the fundamental theorems about spectral synthesis in this field is due to Laurent Schwartz. Recently, several new results on spectral analysis and spectral synthesis have been found on discrete Abelian groups (see [6], [7], [5]) and also in the nondiscrete case (see, e.g., [3], [8]-[10]). In [2], the author formulated problems and proved results concerning spectral synthesis on locally compact Abelian groups. Recently, we introduced a method of studying spectral synthesis problems using annihilators of varieties on locally compact Abelian groups (see [11]). Based on these investigations, it has turned out that the powers of maximal ideals in the group algebra of a discrete Abelian group, or, more generally, in the

Copyright 2016 by the Tusi Mathematical Research Group.

Received Apr. 14, 2015; Accepted Jul. 11, 2015.

2010 Mathematics Subject Classification. Primary 43A25; Secondary 43A45, 22 D15.

Keywords. group algebra, spectral analysis, spectral synthesis, annihilator, exponential monomial. 


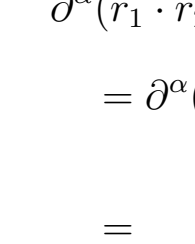

Banach J. Math. Anal. 10 (2016), no. 2, 385-399

http://dx.doi.org/10.1215/17358787-3495693

ISSN: $1735-8787$ (electronic)

http://projecteuclid.org/bjma

\title{
ON THE POWERS OF MAXIMAL IDEALS IN THE MEASURE ALGEBRA
}

\author{
LÁSZLÓ SZÉKELYHIDI \\ Communicated by C. Badea
}

\begin{abstract}
In this paper, we describe the powers of maximal ideals in the measure algebra of some locally compact Abelian groups in terms of the derivatives of the Fourier-Laplace transform of compactly supported measures. We show that if the locally compact Abelian group has sufficiently many real characters, then all derivatives of the Fourier-Laplace transform of a measure at some point of its spectrum completely characterize the measure. We also show that the derivatives of the Fourier-Laplace transform of a measure can be used to describe the powers of the maximal ideals corresponding to the points of the spectrum of the measure on discrete Abelian groups with finite torsion-free rank.
\end{abstract}

\section{INTRODUCTION}

Spectral analysis and spectral synthesis deal with the description of different varieties. One of the fundamental theorems about spectral synthesis in this field is due to Laurent Schwartz. Recently, several new results on spectral analysis and spectral synthesis have been found on discrete Abelian groups (see [6], [7], [5]) and also in the nondiscrete case (see, e.g., [3], [8]-[10]). In [2], the author formulated problems and proved results concerning spectral synthesis on locally compact Abelian groups. Recently, we introduced a method of studying spectral synthesis problems using annihilators of varieties on locally compact Abelian groups (see [11]). Based on these investigations, it has turned out that the powers of maximal ideals in the group algebra of a discrete Abelian group, or, more generally, in the

Copyright 2016 by the Tusi Mathematical Research Group.

Received Apr. 14, 2015; Accepted Jul. 11, 2015.

2010 Mathematics Subject Classification. Primary 43A25; Secondary 43A45, 22 D15.

Keywords. group algebra, spectral analysis, spectral synthesis, annihilator, exponential monomial. 


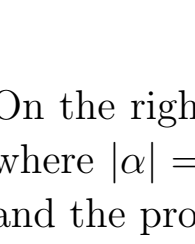

Banach J. Math. Anal. 10 (2016), no. 2, 385-399

http://dx.doi.org/10.1215/17358787-3495693

ISSN: $1735-8787$ (electronic)

http://projecteuclid.org/bjma

\title{
ON THE POWERS OF MAXIMAL IDEALS IN THE MEASURE ALGEBRA
}

\author{
LÁSZLÓ SZÉKELYHIDI \\ Communicated by C. Badea
}

\begin{abstract}
In this paper, we describe the powers of maximal ideals in the measure algebra of some locally compact Abelian groups in terms of the derivatives of the Fourier-Laplace transform of compactly supported measures. We show that if the locally compact Abelian group has sufficiently many real characters, then all derivatives of the Fourier-Laplace transform of a measure at some point of its spectrum completely characterize the measure. We also show that the derivatives of the Fourier-Laplace transform of a measure can be used to describe the powers of the maximal ideals corresponding to the points of the spectrum of the measure on discrete Abelian groups with finite torsion-free rank.
\end{abstract}

\section{INTRODUCTION}

Spectral analysis and spectral synthesis deal with the description of different varieties. One of the fundamental theorems about spectral synthesis in this field is due to Laurent Schwartz. Recently, several new results on spectral analysis and spectral synthesis have been found on discrete Abelian groups (see [6], [7], [5]) and also in the nondiscrete case (see, e.g., [3], [8]-[10]). In [2], the author formulated problems and proved results concerning spectral synthesis on locally compact Abelian groups. Recently, we introduced a method of studying spectral synthesis problems using annihilators of varieties on locally compact Abelian groups (see [11]). Based on these investigations, it has turned out that the powers of maximal ideals in the group algebra of a discrete Abelian group, or, more generally, in the

Copyright 2016 by the Tusi Mathematical Research Group.

Received Apr. 14, 2015; Accepted Jul. 11, 2015.

2010 Mathematics Subject Classification. Primary 43A25; Secondary 43A45, 22 D15.

Keywords. group algebra, spectral analysis, spectral synthesis, annihilator, exponential monomial. 


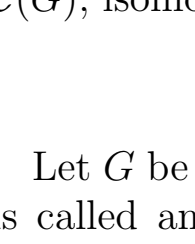

Banach J. Math. Anal. 10 (2016), no. 2, 385-399

http://dx.doi.org/10.1215/17358787-3495693

ISSN: $1735-8787$ (electronic)

http://projecteuclid.org/bjma

\title{
ON THE POWERS OF MAXIMAL IDEALS IN THE MEASURE ALGEBRA
}

\author{
LÁSZLÓ SZÉKELYHIDI \\ Communicated by C. Badea
}

\begin{abstract}
In this paper, we describe the powers of maximal ideals in the measure algebra of some locally compact Abelian groups in terms of the derivatives of the Fourier-Laplace transform of compactly supported measures. We show that if the locally compact Abelian group has sufficiently many real characters, then all derivatives of the Fourier-Laplace transform of a measure at some point of its spectrum completely characterize the measure. We also show that the derivatives of the Fourier-Laplace transform of a measure can be used to describe the powers of the maximal ideals corresponding to the points of the spectrum of the measure on discrete Abelian groups with finite torsion-free rank.
\end{abstract}

\section{INTRODUCTION}

Spectral analysis and spectral synthesis deal with the description of different varieties. One of the fundamental theorems about spectral synthesis in this field is due to Laurent Schwartz. Recently, several new results on spectral analysis and spectral synthesis have been found on discrete Abelian groups (see [6], [7], [5]) and also in the nondiscrete case (see, e.g., [3], [8]-[10]). In [2], the author formulated problems and proved results concerning spectral synthesis on locally compact Abelian groups. Recently, we introduced a method of studying spectral synthesis problems using annihilators of varieties on locally compact Abelian groups (see [11]). Based on these investigations, it has turned out that the powers of maximal ideals in the group algebra of a discrete Abelian group, or, more generally, in the

Copyright 2016 by the Tusi Mathematical Research Group.

Received Apr. 14, 2015; Accepted Jul. 11, 2015.

2010 Mathematics Subject Classification. Primary 43A25; Secondary 43A45, 22 D15.

Keywords. group algebra, spectral analysis, spectral synthesis, annihilator, exponential monomial. 


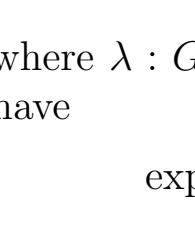

Banach J. Math. Anal. 10 (2016), no. 2, 385-399

http://dx.doi.org/10.1215/17358787-3495693

ISSN: $1735-8787$ (electronic)

http://projecteuclid.org/bjma

\title{
ON THE POWERS OF MAXIMAL IDEALS IN THE MEASURE ALGEBRA
}

\author{
LÁSZLÓ SZÉKELYHIDI \\ Communicated by C. Badea
}

\begin{abstract}
In this paper, we describe the powers of maximal ideals in the measure algebra of some locally compact Abelian groups in terms of the derivatives of the Fourier-Laplace transform of compactly supported measures. We show that if the locally compact Abelian group has sufficiently many real characters, then all derivatives of the Fourier-Laplace transform of a measure at some point of its spectrum completely characterize the measure. We also show that the derivatives of the Fourier-Laplace transform of a measure can be used to describe the powers of the maximal ideals corresponding to the points of the spectrum of the measure on discrete Abelian groups with finite torsion-free rank.
\end{abstract}

\section{INTRODUCTION}

Spectral analysis and spectral synthesis deal with the description of different varieties. One of the fundamental theorems about spectral synthesis in this field is due to Laurent Schwartz. Recently, several new results on spectral analysis and spectral synthesis have been found on discrete Abelian groups (see [6], [7], [5]) and also in the nondiscrete case (see, e.g., [3], [8]-[10]). In [2], the author formulated problems and proved results concerning spectral synthesis on locally compact Abelian groups. Recently, we introduced a method of studying spectral synthesis problems using annihilators of varieties on locally compact Abelian groups (see [11]). Based on these investigations, it has turned out that the powers of maximal ideals in the group algebra of a discrete Abelian group, or, more generally, in the

Copyright 2016 by the Tusi Mathematical Research Group.

Received Apr. 14, 2015; Accepted Jul. 11, 2015.

2010 Mathematics Subject Classification. Primary 43A25; Secondary 43A45, 22 D15.

Keywords. group algebra, spectral analysis, spectral synthesis, annihilator, exponential monomial. 


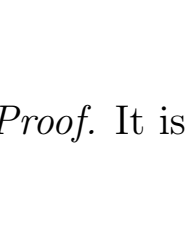

Banach J. Math. Anal. 10 (2016), no. 2, 385-399

http://dx.doi.org/10.1215/17358787-3495693

ISSN: $1735-8787$ (electronic)

http://projecteuclid.org/bjma

\title{
ON THE POWERS OF MAXIMAL IDEALS IN THE MEASURE ALGEBRA
}

\author{
LÁSZLÓ SZÉKELYHIDI \\ Communicated by C. Badea
}

\begin{abstract}
In this paper, we describe the powers of maximal ideals in the measure algebra of some locally compact Abelian groups in terms of the derivatives of the Fourier-Laplace transform of compactly supported measures. We show that if the locally compact Abelian group has sufficiently many real characters, then all derivatives of the Fourier-Laplace transform of a measure at some point of its spectrum completely characterize the measure. We also show that the derivatives of the Fourier-Laplace transform of a measure can be used to describe the powers of the maximal ideals corresponding to the points of the spectrum of the measure on discrete Abelian groups with finite torsion-free rank.
\end{abstract}

\section{INTRODUCTION}

Spectral analysis and spectral synthesis deal with the description of different varieties. One of the fundamental theorems about spectral synthesis in this field is due to Laurent Schwartz. Recently, several new results on spectral analysis and spectral synthesis have been found on discrete Abelian groups (see [6], [7], [5]) and also in the nondiscrete case (see, e.g., [3], [8]-[10]). In [2], the author formulated problems and proved results concerning spectral synthesis on locally compact Abelian groups. Recently, we introduced a method of studying spectral synthesis problems using annihilators of varieties on locally compact Abelian groups (see [11]). Based on these investigations, it has turned out that the powers of maximal ideals in the group algebra of a discrete Abelian group, or, more generally, in the

Copyright 2016 by the Tusi Mathematical Research Group.

Received Apr. 14, 2015; Accepted Jul. 11, 2015.

2010 Mathematics Subject Classification. Primary 43A25; Secondary 43A45, 22 D15.

Keywords. group algebra, spectral analysis, spectral synthesis, annihilator, exponential monomial. 


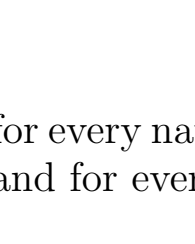

Banach J. Math. Anal. 10 (2016), no. 2, 385-399

http://dx.doi.org/10.1215/17358787-3495693

ISSN: $1735-8787$ (electronic)

http://projecteuclid.org/bjma

\title{
ON THE POWERS OF MAXIMAL IDEALS IN THE MEASURE ALGEBRA
}

\author{
LÁSZLÓ SZÉKELYHIDI \\ Communicated by C. Badea
}

\begin{abstract}
In this paper, we describe the powers of maximal ideals in the measure algebra of some locally compact Abelian groups in terms of the derivatives of the Fourier-Laplace transform of compactly supported measures. We show that if the locally compact Abelian group has sufficiently many real characters, then all derivatives of the Fourier-Laplace transform of a measure at some point of its spectrum completely characterize the measure. We also show that the derivatives of the Fourier-Laplace transform of a measure can be used to describe the powers of the maximal ideals corresponding to the points of the spectrum of the measure on discrete Abelian groups with finite torsion-free rank.
\end{abstract}

\section{INTRODUCTION}

Spectral analysis and spectral synthesis deal with the description of different varieties. One of the fundamental theorems about spectral synthesis in this field is due to Laurent Schwartz. Recently, several new results on spectral analysis and spectral synthesis have been found on discrete Abelian groups (see [6], [7], [5]) and also in the nondiscrete case (see, e.g., [3], [8]-[10]). In [2], the author formulated problems and proved results concerning spectral synthesis on locally compact Abelian groups. Recently, we introduced a method of studying spectral synthesis problems using annihilators of varieties on locally compact Abelian groups (see [11]). Based on these investigations, it has turned out that the powers of maximal ideals in the group algebra of a discrete Abelian group, or, more generally, in the

Copyright 2016 by the Tusi Mathematical Research Group.

Received Apr. 14, 2015; Accepted Jul. 11, 2015.

2010 Mathematics Subject Classification. Primary 43A25; Secondary 43A45, 22 D15.

Keywords. group algebra, spectral analysis, spectral synthesis, annihilator, exponential monomial. 


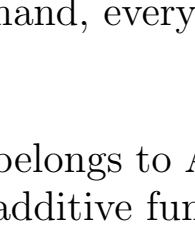

Banach J. Math. Anal. 10 (2016), no. 2, 385-399

http://dx.doi.org/10.1215/17358787-3495693

ISSN: $1735-8787$ (electronic)

http://projecteuclid.org/bjma

\title{
ON THE POWERS OF MAXIMAL IDEALS IN THE MEASURE ALGEBRA
}

\author{
LÁSZLÓ SZÉKELYHIDI \\ Communicated by C. Badea
}

\begin{abstract}
In this paper, we describe the powers of maximal ideals in the measure algebra of some locally compact Abelian groups in terms of the derivatives of the Fourier-Laplace transform of compactly supported measures. We show that if the locally compact Abelian group has sufficiently many real characters, then all derivatives of the Fourier-Laplace transform of a measure at some point of its spectrum completely characterize the measure. We also show that the derivatives of the Fourier-Laplace transform of a measure can be used to describe the powers of the maximal ideals corresponding to the points of the spectrum of the measure on discrete Abelian groups with finite torsion-free rank.
\end{abstract}

\section{INTRODUCTION}

Spectral analysis and spectral synthesis deal with the description of different varieties. One of the fundamental theorems about spectral synthesis in this field is due to Laurent Schwartz. Recently, several new results on spectral analysis and spectral synthesis have been found on discrete Abelian groups (see [6], [7], [5]) and also in the nondiscrete case (see, e.g., [3], [8]-[10]). In [2], the author formulated problems and proved results concerning spectral synthesis on locally compact Abelian groups. Recently, we introduced a method of studying spectral synthesis problems using annihilators of varieties on locally compact Abelian groups (see [11]). Based on these investigations, it has turned out that the powers of maximal ideals in the group algebra of a discrete Abelian group, or, more generally, in the

Copyright 2016 by the Tusi Mathematical Research Group.

Received Apr. 14, 2015; Accepted Jul. 11, 2015.

2010 Mathematics Subject Classification. Primary 43A25; Secondary 43A45, 22 D15.

Keywords. group algebra, spectral analysis, spectral synthesis, annihilator, exponential monomial. 


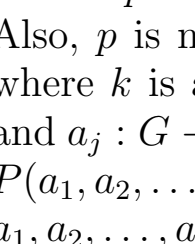

Banach J. Math. Anal. 10 (2016), no. 2, 385-399

http://dx.doi.org/10.1215/17358787-3495693

ISSN: $1735-8787$ (electronic)

http://projecteuclid.org/bjma

\title{
ON THE POWERS OF MAXIMAL IDEALS IN THE MEASURE ALGEBRA
}

\author{
LÁSZLÓ SZÉKELYHIDI \\ Communicated by C. Badea
}

\begin{abstract}
In this paper, we describe the powers of maximal ideals in the measure algebra of some locally compact Abelian groups in terms of the derivatives of the Fourier-Laplace transform of compactly supported measures. We show that if the locally compact Abelian group has sufficiently many real characters, then all derivatives of the Fourier-Laplace transform of a measure at some point of its spectrum completely characterize the measure. We also show that the derivatives of the Fourier-Laplace transform of a measure can be used to describe the powers of the maximal ideals corresponding to the points of the spectrum of the measure on discrete Abelian groups with finite torsion-free rank.
\end{abstract}

\section{INTRODUCTION}

Spectral analysis and spectral synthesis deal with the description of different varieties. One of the fundamental theorems about spectral synthesis in this field is due to Laurent Schwartz. Recently, several new results on spectral analysis and spectral synthesis have been found on discrete Abelian groups (see [6], [7], [5]) and also in the nondiscrete case (see, e.g., [3], [8]-[10]). In [2], the author formulated problems and proved results concerning spectral synthesis on locally compact Abelian groups. Recently, we introduced a method of studying spectral synthesis problems using annihilators of varieties on locally compact Abelian groups (see [11]). Based on these investigations, it has turned out that the powers of maximal ideals in the group algebra of a discrete Abelian group, or, more generally, in the

Copyright 2016 by the Tusi Mathematical Research Group.

Received Apr. 14, 2015; Accepted Jul. 11, 2015.

2010 Mathematics Subject Classification. Primary 43A25; Secondary 43A45, 22 D15.

Keywords. group algebra, spectral analysis, spectral synthesis, annihilator, exponential monomial. 